Journal of Integrative Neuroscience, Vol. 6, No. 3 (2007) 1-4

(c) Imperial College Press

\title{
EDITORIAL
}

\section{MULTISENSORY INTEGRATION AND PARALLEL MEMORY SYSTEMS FOR SPATIAL COGNITION}

The large body of research devoted to the neural bases of spatial memory has unveiled the need to study numerous subfunctions including: (i) neural information processing and coding (e.g., information storage and transmission at synapses); (ii) multisensory integration (e.g., extraction of low-dimensional representations from high-dimensional sensory flows; combination of multimodal signals into unified percepts); (iii) procedural memory (e.g., acquisition of low-level sensory-motor couplings; fine tuning of coordinate movements adapted to the sensory-motor context); (iv) declarative memory (e.g., elaboration of abstract descriptions of the spatiotemporal relationships between spatial events); (v) action selection (e.g., reward-dependent navigation planning; dynamical weighing of multiple concurrent strategies).

In addition to this broad functional spectrum, understanding spatial memory requires a cross linking of different description levels including: (i) the molecular level (e.g., dynamics of gene expression in normal and mutant organisms; dynamics of the molecular influx through receptor channels; neuropharmacological modulation of neural states); (ii) the synaptic level (e.g., mechanisms regulating the presynaptic neurotransmitter release; biophysics of the transduction of presynaptic spikes into analog postsynaptic potentials; activity-dependent synaptic plasticity providing the basis for spatial learning and memory); (iii) the cellular level (e.g., electrophysiological properties of single neurons; mechanisms regulating the intrinsic electroresponsiveness of nerve cells; temporal dynamics of the emitted spike trains); (iv) the local network level (e.g., mechanisms underlying the emergence of neural population states; population activity dynamics for encoding, storage, and retrieval of spatial information); (v) the system level (e.g., anatomo-functional interactions of multiple brain structures mediating spatial learning functions); (vi) the behavioral level (e.g., learning mechanisms promoting flexible solutions to solve complex navigation tasks; phylogenetic comparisons and ontogenetic development of spatial learning capabilities).

Integrative neuroscience provides a suitable framework to study wide horizontal function spectra as well as vertical interactions across different levels of organization. This approach promotes an interdisciplinary effort to combine and integrate findings from experimental neuroscience (e.g., molecular, electrophysiological, and 
behavioral studies), theoretical neuroscience (e.g., physics, mathematics), and neuroengineering (e.g., neuroinformatics, neurorobotics). For instance, theoretical models offer a unique vantage point from which to derive predictions that can be tested in innovative experiments. Mathematical representations permit to describe both the space and time components characterizing the couplings between neurobiological processes. Thus, theory can allow one to scale up from the biophysical properties of single cells to the dynamics of large neural populations, organize them in subsystems, and generate hypotheses about their spatiotemporal interactions. Still, likewise experimental observations at different levels can be difficult to relate to each other, formal models that merely apply to specific mechanisms without sharing a common theoretical framework can make it difficult to derive integrated descriptions. As a consequence, today's efforts in theoretical neurobiology aim at developing a general theory accounting for multiple abstraction levels and favoring the integration of a variety of formal descriptions into a comprehensive view (see, for instance, Chauvet [1]).

This special issue of the Journal of Integrative Neuroscience arose from a workshop held at the University La Sapienza in Rome, in 2006, in the frame of the International Conference on the Simulation of Adaptive Behavior. Attendees at the workshop included experimentalists, theoretical neuroscientists, and roboticists, who focused on the multiple phenomena related to spatial learning and navigation. Two main questions were prominently addressed by the invited speakers and the poster presentations:

- what are the learning mechanisms permitting the integration of multisensory signals into spatial memories?

- what are the cooperative/competitive interrelations between the neural substrates (e.g., hippocampus, prefrontal and parietal cortices, basal ganglia, and cerebellum) mediating multiple spatial memory systems?

The compiled series of papers presents a cross-disciplinary work — ranging from electrophysiological and behavioral studies to mathematical modeling and robotic implementations - to investigate the manifold nature of spatial cognition. One review article and six original papers are presented: two experimental reports, two modeling works, and two robotic implementations.

The review paper by Arleo and Rondi-Reig sets the scene by surveying the wealth of research on spatial cognition and navigation, with particular emphasis on the neuronal mechanisms and decision making processes that regulate the selection of navigation strategies. In addition to reviewing results obtained from animal experiments - including molecular, electrophysiological and behavioral studies the paper introduces the neurocomputational approach behind both modeling and neurorobotic work on spatial learning and navigation.

The experimental contribution by Hok et al. concentrates on the properties of hippocampal place cells, pyramidal neurons that discharge selectively according to the spatial location of an animal. In this work, electrophysiological recordings are 
performed in the CA1 dorsal hippocampus of freely moving rats. The goal-related responses of CA1 cells are investigated by means of two behavioral navigation tasks. The reported data confirm previous authors' findings [4], and extend them by showing that place cell discharges can encode the time prediction of forthcoming reward delivery. The authors also discuss their results in light of the current hippocampal theories, in particular, regarding the role of the hippocampus in sequence learning.

The next paper, by Lambrey and Berthoz, presents a new series of human experiments focusing on the gender differences in the use of spatial information, namely external landmarks versus self-motion cues. Subjects were asked to learn a pointing behavioral task under multiple experimental conditions - different in terms of reliability of external landmarks. The reported findings suggest that men tended to employ spatial representations updated through the integration of self-motion signals, whereas women seemed to mainly rely upon the information provided by (presumed stable) external landmarks. This data add to the current literature (see, for instance, the review by Loomis et al. [6]) suggesting that the learning of metric spatial information is more accurate in men than in women, whereas the representation of landmark information is richer in women than in men.

Fleischer and Krichmar study the influence of different sensory modalities onto the spatial coding in the hippocampus. By means of a neuromimetic model of the rodent's medial temporal lobe embodied in a robotic device, they investigate the effect of impairing different sensory pathways on the stability of hippocampal place field representations. They show that no single lesion has significant effects on both the behavioral performance and the hippocampal spatial coding. This result confirms, by means of a systematic and quantitative analysis, that the spatial selectivity of hippocampal neurons is the result of a multimodal process that integrates information from numerous sensory sources (see Knierim et al. [5], for a recent review). Moreover, a causality analysis is presented that shows how different neural pathways may lead to the same place cell activity, with a greater variability on entorhinal cortex or sensory coding neurons.

Recently, research on spatial cognition has been largely influenced by the discovery of "grid cells" in the rat medial entorhinal cortex [3]. In contrast to hippocampal place cells, these spatially selective neurons are highly active at multiple regions arranged in regular triangular grids. In this special issue, Guanella and Verschure analyze the key properties of grid cell populations based on theoretical simulations. Their work provide a mathematical demonstration of how these neurons encode spatial information in a compact and robust manner, which generates relevant insights for high level modeling of spatial coding.

The paper by Gaussier et al. is also devoted to grid cells, but the emphasis is put on a high-level spatial learning architecture suitable for a robotic implementation. In the presented model, grid cell activity is driven by a path integration system mediated by the retrosplenial and/or parietal cortices. It is shown that the information provided by the grid cell discharges can be prominent to yield precise and robust place cell responses, even in the absence of visual inputs. The presented 
model extends the authors' previous hypothesis that hippocampal place cell activity would code transitions between two locations rather than mere spatial positions [2].

Finally, the contribution by Debaecker and Benosman presents a neuromimetic model of early visual information processing - at the level of the retina and of the lateral geniculate nucleus. The authors propose a mathematical model of early feature detection for the recognition of visual scenes, and they focus on the robustness of the method to different classes of noise. The model accounts for the spatial distribution of retinal photoreceptors and exploits it to generate a low-dimensional decomposition of the images based on local Gaussian color histograms. It is also shown how this visual encoding process can be relevant to spatial localization.

\section{ANGELO ARLEO and RICARDO CHAVARRIAGA Guest Editors}

\section{References}

[1] Chauvet GA, A new paradigm for theory in integrative biology: The principle of autoassociative stabilization: Biochemical networks and the selection of neuronal groups, J Integr Neurosci 5:381-415, 2006.

[2] Gaussier P, Revel A, Banquet JP, Babeau V, From view cells and place cells to cognitive map learning: Processing stages of the hippocampal system, Biol Cybern 86:15-28, 2002.

[3] Hafting T, Fyhn M, Molden S, Moser MB, Moser EI, Microstructure of a spatial map in the entorhinal cortex, Nature 436:801-806, 2005.

[4] Hok V, Lenck-Santini PP, Roux S, Save E, Muller RU, Poucet B, Goal-related activity in hippocampal place cells, J Neurosci 27:472-482, 2007.

[5] Knierim JJ, Lee I, Hargreaves EL, Hippocampal place cells: Parallel input streams, subregional processing, and implications for episodic memory, Hippocampus 16:755-764, 2006.

[6] Loomis JM, Klatzky RL, Golledge RG, Philbeck JW, Human navigation by path integration, in Golledge RG (ed.), Wayfinding Behavior, The Johns Hopkins University Press, pp. $125-151,1999$. 